\title{
Left anterior hemiblock masking coronary insufficiency
}

\author{
ULDERICO VOLPE
}

From the Department of Clinical Physiology, Danderyd Hospital, Danderyd, Sweden

SUMMARY A case is presented in which transient left anterior hemiblock masked the electrocardiographic signs of coronary insufficiency during work.

There is a well-documented observation that left anterior hemiblock is capable of concealing the electrocardiographic signs of inferior and anteroseptal infarction, but no case has been reported that shows left anterior hemiblock concealing the evidence of coronary insufficiency during exercise. The purpose of this communication is to report such a case.

\section{Case report}

A 58-year-old man was referred to our physiological laboratory for an exercise test. The patient was known to have diabetes and pain in the left shoulder on exertion suggesting the diagnosis of angina pectoris. The electrocardiogram at rest showed signs of left ventricular hypertrophy with ST segment depression of $1 \mathrm{~mm}$ in leads II, III, aVF, and CR 4-7 (Fig. 1). The patient performed a standardised exercise test in the upright position on a bicycle ergometer with the electrocardiographic recording from bipolar chest-head leads $(\mathrm{CH})$. The exercise started at 50 Watts but was stopped after 2 minutes because of a short bout of ventricular tachycardia and an intermittent ventricular block with prominent $S$ waves in $\mathrm{CH}$ 5-7. After a rest of 10 minutes the exercise was reinitiated, this time at 40 Watts with the praecordial leads as before, and with one electrode over the left clavicle, a second electrode over the right clavicle, and a third over L5 in order to record the standard leads. During work the patient experienced pain in the left shoulder and at the same time the ST segment depressed $2 \mathrm{~mm}$ in lead $\mathrm{CH} 3-7$, and once again a transient block developed with changes in the electrocardiogram consistent with left anterior hemiblock. Each time the left anterior hemiblock appeared the ST depression was lost, with a return of this electrocardiographic evidence of coronary insufficiency as soon as the ventricular conduction returned to normal (Fig. 2). After work the prae- cordial leads CR 3-7 showed a downsloping ST depressed segment (Fig. 3).

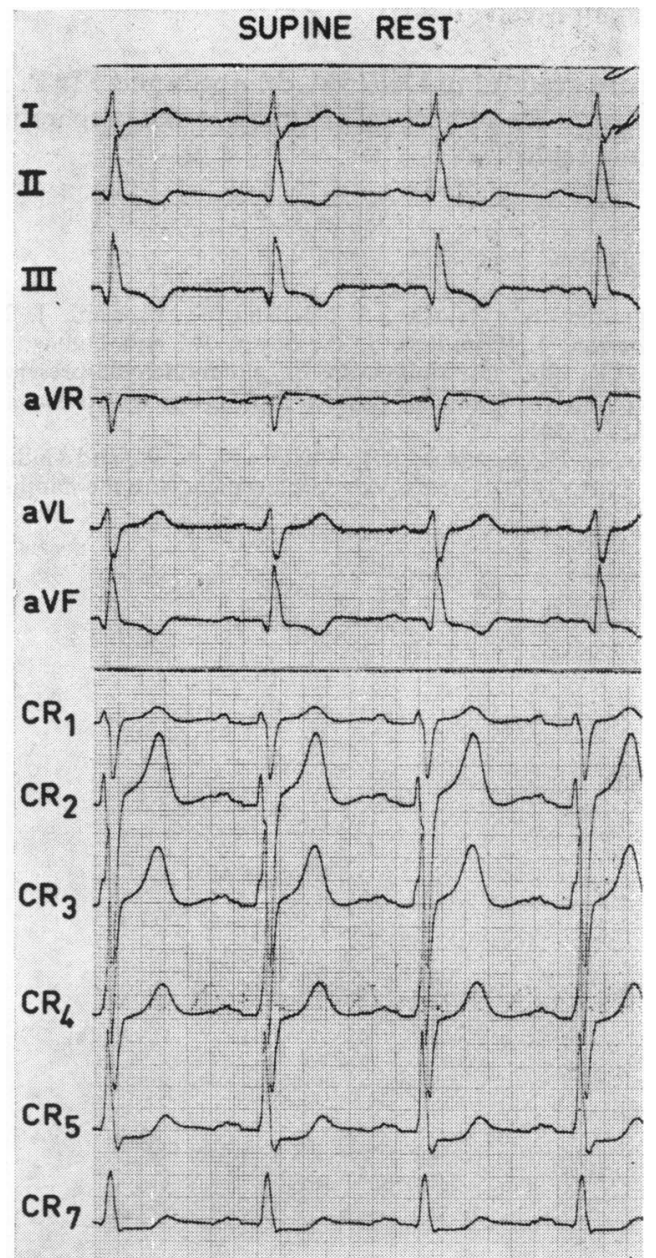

Fig. 1 Electrocardiogram at rest with evidence of left ventricular hypertrophy and slight depression of the $S T$ segment. 


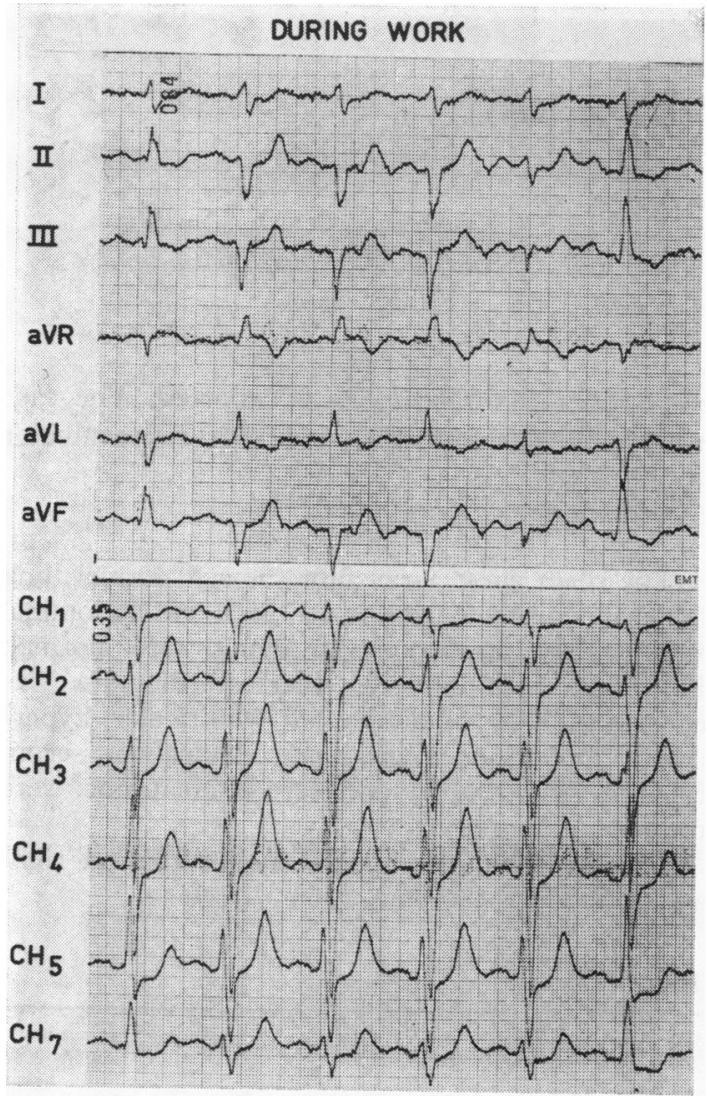

Fig. 2 Electrocardiogram during work showing in the first and the last beat a more pronounced ST depression. The second, the third, the fourth and the fifth beat show the features of left anterior hemiblock and the return to normal of the ST segment.

\section{Discussion}

Rosenbaum et al. (1972) have pointed out that left anterior hemiblock may obscure the electrocardiographic signs of inferior myocardial infarction. Recently Cristal et al. (1975) reported a case with anatomical and pathological findings that confirmed the hypothesis of Rosenbaum, stating that this diagnostic difficulty presupposes an intact left posterior papillary muscle. Altieri and Schaal (1973) similarly reported a case where left anterior hemiblock masked the electrocardiographic evidence of inferior and anteroseptal myocardial infarction.

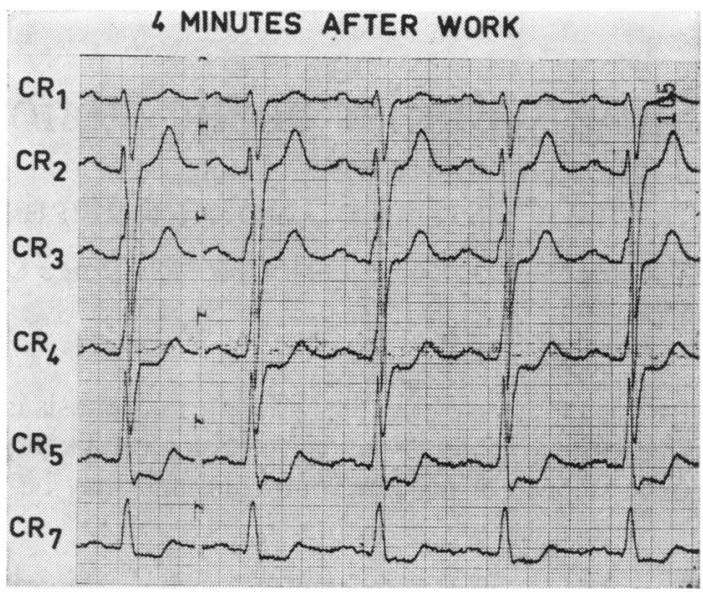

Fig. 3 The praecordial leads after work showing downward sloping depression of the ST segment in CR 4-7 consistent with coronary insufficiency.

The cause of the change in repolarisation in the present case is uncertain. It is possible, however, that in this patient the normal and late repolarisation of the anterior and superior wall of the left ventricle counterbalanced the vector of ischaemia originating from the lateral and inferior wall of the left ventricle. This case emphasises the need for a careful evaluation of the electrocardiographic reaction to exercise in patients with left anterior hemiblock and suspected angina, since the diagnosis of coronary insufficiency can be impossible to show unless the hemiblock is transient.

\section{References}

Altieri, P., and Schaal, S. F. (1973). Inferior and anteroseptal myocardial infarction concealed by transient left anterior hemiblock. Fournal of Electrocardiology, 6, 257-258.

Cristal, N., Ho, W., and Gueron, M. (1975). Left anterior hemiblock masking inferior myocardial infarction. British Heart fournal, 37, 543-547.

Rosenbaum, M. B., Elizari, M. V., Lazzari, J. O., Nau, G. S., Halpern, M. S., and Levi, R. J. (1972). The differential electrocardiographic manifestations of hemiblocks, bilateral bundle branch block, and trifascicular blocks. In Advances in Electrocardiography, pp. 145-182. Ed. by R. C. Schlant and J. W. Hurst. Grune and Stratton, New York.

Requests for reprints to Dr Ulderico Volpe, Department of Clinical Physiology, Danderyd Hospital, S-182 03 Danderyd, Sweden. 